\title{
Effect of Electronic Cigarettes on Smoking Reduction and Cessation in Korean Male Smokers: A Randomized Controlled Study
}

\author{
Seung-Hwa Lee, MD, Sang-Hyun Abn, and Yoo-Seock Cheong, MD, PhD
}

Introduction: New approaches to electronic cigarettes (e-cigarettes) as a form of nicotine replacement therapy (NRT) may reduce the rates of tobacco-related disease and mortality. Therefore, we investigated the effect of e-cigarettes on smoking cessation compared with nicotine gum.

Methods: A total of 150 subjects were randomly assigned to 2 groups and each was allocated a 12week supply of either e-cigarettes or nicotine gum. The continuous abstinence rate, 7-day point prevalence of abstinence, smoking reduction rate and amount, and tolerability were evaluated.

Results: There were no statistically significant differences in the effectiveness-related parameters of smoking cessation, such as 9- to 12-week, 9- to 24-week, and 12- and 24-week point prevalence of abstinence, between the 2 groups. However, although the reduction in cigarette smoking was similar, the proportion of subjects who showed smoking reduction at 24 weeks was higher in the e-cigarette group than the nicotine gum group. In addition, adverse events were significantly less frequent in the e-cigarette group than in the nicotine gum group.

Conclusions: In our study, the effect of e-cigarettes on smoking cessation was similar compared with that of nicotine gum, a well-documented NRT. In addition, e-cigarettes were well tolerated by the study population. Therefore, the use of e-cigarettes as an NRT may be considered for smoking-cessation purposes. A large-scale prospective randomized controlled trial is necessary to clarify our results. ( $\mathrm{J}$ Am Board Fam Med 2019;32:567-574.)

Keywords: Cigarette Smoking, Electronic Nicotine Delivery Systems, Korea, Nicotine Chewing Gum, Smoking Cessation, Smoking Reduction, Tobacco Products

The electronic cigarette (e-cigarette) is a batterypowered device that delivers nicotine in a warm vapor. ${ }^{1,2}$ It seems and feels similar to a traditional

This article was externally peer reviewed.

Submitted 24 December 2018; revised 28 March 2019; accepted 1 April 2019.

From Department of Family Medicine, Seohae Hospital, Seocheon, Republic of Korea (S-HL); Department of Family Practice and Community Health, Smoking Cessation Center, Ajou University School of Medicine, Suwon, Republic of Korea (S-HL); Department of Family Medicine, Seoul National University Hospital, Seoul, Republic of Korea (S-HA); Department of Family Medicine, Smoking Cessation Center, Dankook University College of Medicine, Cheonan, Republic of Korea (Y-SC).

Funding: none.

Conflict of interest: none declared.

Corresponding author: Yoo-Seock Cheong, MD, PhD, Department of Family Medicine, Smoking Cessation Center, Dankook University College of Medicine, 201 Manghyangro, Dongnam-gu, Cheonan, Chungcheongnam-do, 31116 Republic of Korea (E-mail: fmdoctor@hanmail.net).

${ }^{*} \mathrm{~S}-\mathrm{H}$ Lee and S-HA contributed equally to this work. combustible cigarette. In its third report on the scientific basis of tobacco-product regulation, the World Health Organization (WHO) defined e-cigarettes as electronic nicotine delivery systems. ${ }^{3,4}$ E-cigarette companies advertise their products as being effective for smoking cessation or reduction and ecologically friendly. ${ }^{5}$ A recent article found that although the long-term effects of e-cigarette use are not known, these devices are probably safer than combustible tobacco products. ${ }^{6}$ Indeed, most people who purchase e-cigarettes report doing so as part of an attempt to quit smoking altogether or to reduce their combustible cigarette consumption. ${ }^{3,5}$ Thus, e-cigarettes may be considered to be lower risk substitutes for traditional cigarettes. ${ }^{7}$ Moreover, new approaches to e-cigarettes as a nicotine replacement therapy (NRT) and as fast-acting nicotine inhalation devices may help to reduce the burden of tobacco-related disease and mortality in 
current smokers. However, the efficacy of the ecigarette as an aid in smoking cessation or reduction is still controversial. Few studies have focused on the relationship between e-cigarette use and smoking cessation. ${ }^{8-12}$ Therefore, the purpose of this study was to evaluate the effectiveness of e-cigarettes in smoking cessation compared with that of nicotine gum, a well-documented NRT.

\section{Methods}

\section{Study Population}

This study was a single-center, prospective, openlabel, randomized controlled, clinical pilot trial. From January to September 2012, 218 Korean male adults over 18 years of age were recruited from a motor company in Cheonan, Republic of Korea. Because most employees of this company were male, we recruited only male employees in the study. Eligible subjects had smoked at least 10 cigarettes per day during the preceding year, had smoked for at least 3 years, and were motivated to stop smoking entirely or to reduce their cigarette consumption. Among the initial 218 subjects, 68 were excluded for the following reasons: (1) declined to participate $(\mathrm{n}=26)$; (2) had a past medical history of serious clinical diseases $(\mathrm{n}=38)$ such as malignancy, cardiovascular disease, cerebrovascular disease, pulmonary disease, and significant hepatic or renal impairment; and (3) had attempted to stop smoking in the past 12 months by using other NRTs $(n=4)$. Finally, 150 participants were included in the study.

\section{Study Design}

The 150 enrolled subjects were randomly assigned to 2 groups according to a computer-generated randomization sequence with a block size of 2 . The e-cigarette (EC) group $(n=75)$ and the nicotine gum $(\mathrm{NG})$ group $(\mathrm{n}=75)$ received e-cigarettes or nicotine gum, respectively. A total of 132 subjects received treatment and completed the study; 4 and 14 subjects originally assigned to the EC and NG groups, respectively, withdrew before treatment. Those 18 subjects who did not receive treatment were included in the analyses on an intention-totreat basis. The enrollment and assignment of all subjects were performed by a clinical research coordinator not involved in the study. All investigators involved in the study were blinded to group allocation. The protocol and study design are presented in Figure 1.

\section{Ethics Statement}

This study was conducted in accordance with the Declaration of Helsinki, ${ }^{13}$ and is consistent with the guidelines of Good Clinical Practice. ${ }^{14}$ The study was approved by the Institutional Review Board of Dankook University Hospital (approval no. 1101-008). In addition, the study was registered with the Clinical Research Information Service, number KCT0001277, the Korean national clinical trial registry in the WHO International Clinical Trials Registry Platform. Written informed consent was obtained from all subjects before enrollment in the study.

\section{Interventions}

Fifty-minute education sessions on smoking cessation and the use of smoking-cessation aids were conducted in the medical office where the e-cigarette and nicotine gum were distributed. At enrollment, we collected subject information, which included age, sex, education, marriage status, alcohol consumption ( $\geq 70 \mathrm{~g} /$ week or $\geq 10 \mathrm{~g} /$ day), exercise (at least once per week on a regular basis), and detailed data regarding cigarette smoking habits (cumulative smoking amount, cigarettes smoked per day, duration of smoking, confidence in the ability to quit smoking, Fagerström test for nicotine dependence, and prior attempts to quit). After completion of a basic questionnaire, follow-up questionnaires were administered at weeks 12 and 24. The subjects were instructed to visit the medical office every 4 weeks for evaluation and counseling by an independent health practitioner. The end-expiratory carbon monoxide (Micro-4 smokerlyzer; Bedfont Scientific Ltd., NJ) and urine cotinine (AccuSign nicotine; Princeton Biomeditceh Co., Princeton, NJ) levels were measured at baseline, and at the final visit (24-week). We used an e-cigarette (eGO-C Ovale, nicotine $0.01 \mathrm{mg} / \mathrm{mL}$; Janty-Korea Co., Janty-Asia Co., Seoul, Republic of Korea) and nicotine gum (Nicoman, nicotine 2 mg/tablet; Daewoong Pharmaceuticals, Seongnam, Republic of Korea) (Figure 2), both of which were distributed in 12-week supplies. 
Figure 1. Consolidated Standards of Reporting Trials flow chart detailing conduct of the study. MHx., medical history; ds., disease; NRT, nicotine replacement therapy; NG, nicotine gum; EC, e-cigarette.

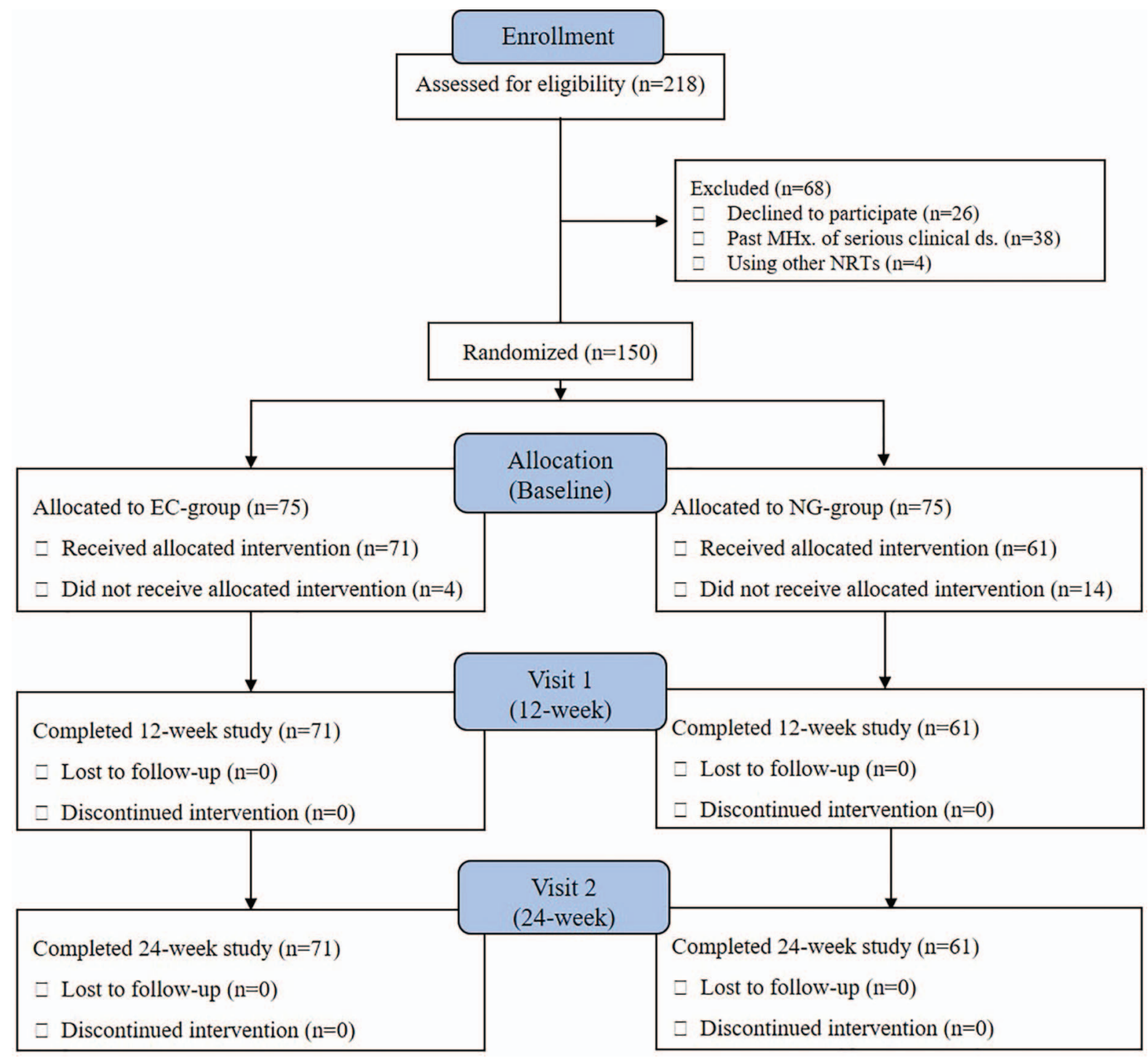

\section{Efficacy Outcomes \\ Primary End Points}

In our study, the primary outcomes were 9- to 12-week and 9- to 24-week continuous abstinence rates (CARs). They were verified not only by selfreported questionnaires but also by means of urine cotinine (negative) and end-expiratory carbon monoxide $(<10 \mathrm{ppm})$ measurements.

\section{Secondary End Points}

The secondary outcomes were the 7-day point prevalence (PP) of abstinence at weeks 12 and 24, an evaluation of the decrease in smoking as an estimation of the harm-reduction effect of e-ciga- rette, and analysis of adverse events (AEs) for tolerability assessment.

\section{Statistical Analysis}

All continuous variables were expressed as means \pm standard deviations and categorical variables as numbers and percentages. Differences in continuous variables between the $\mathrm{EC}$ and $\mathrm{NG}$ groups were analyzed using the independent $t$ test, and categorical variables were analyzed using the $\chi^{2}$ test or Fisher-Freeman-Halton extension of Fisher's probability test, as appropriate. We conducted multivariable logistic regression analyses controlling for possible confounders in both groups. $P$ values less 
Figure 2. Intervention products used in the present study: (A) e-cigarette and cartilage; (B) components of an electronic cigarette, ${ }^{1}$ rechargeable battery, ${ }^{2}$ digital button, ${ }^{3}$ main body, consisting of a controller and an atomizer, ${ }^{4}$ mouthpiece with chamber containing e-cigarette fluid, ${ }^{5}$ and disposable mouthpiece cover; and (C) nicotine gum.
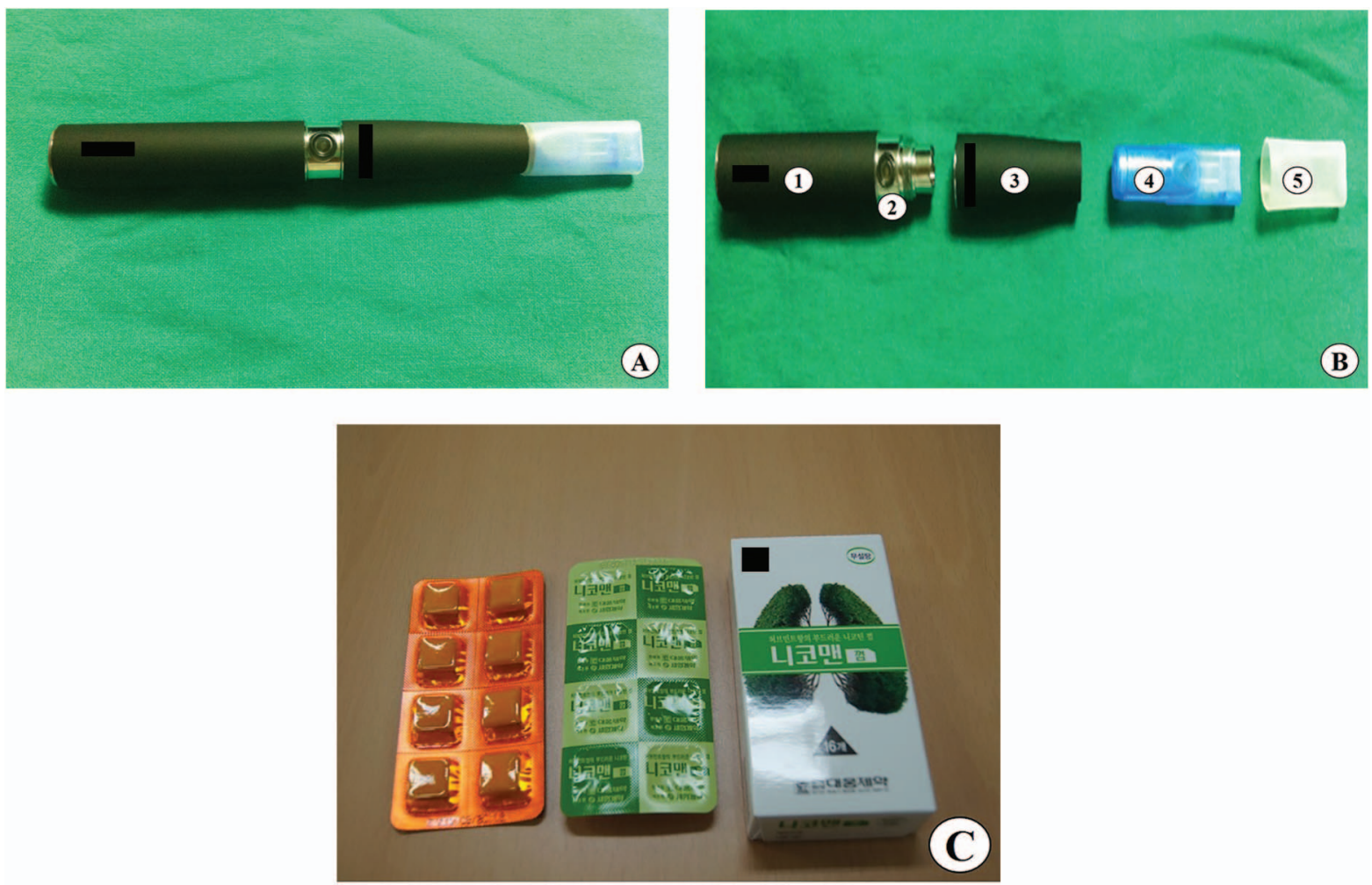

than 0.05 were considered as statistically significant. All statistical analyses were performed using SPSS version 20.0 (SPSS Inc., Chicago, IL).

\section{Results}

\section{Baseline Characteristics of the Study Subjects}

The baseline characteristics of the 2 groups (EC vs NG group) are summarized in Table 1 . The mean age was $42.3 \pm 8.3$ years (range, 24 to 57 ), and the study population consisted of all males (100\%). Overall, $39.3 \%$ of subjects had a college-level education, most $(90 \%)$ were married, $71.3 \%$ were current alcohol users, and $54.7 \%$ were regular exercisers. Because of the inclusion criteria, all were current smokers. Total smoking amount, cigarettes smoked per day, and smoking duration in all subjects were $21.56 \pm 9.71$ pack-years (range, 3 to 50 ), $1.01 \pm 0.37 \mathrm{packs} /$ day (range, 0.5 to 2.0 ), and $21.98 \pm 8.76$ years (range, 4 to 44 ), respectively. Among the participants, $90.0 \%$ reported prior attempts to quit cigarette smoking and only $10 \%$ had never attempted to stop. The Fagerström test for nicotine dependence and initial confidence score were $4.05 \pm 2.24$ and $5.95 \pm 2.67$, out of a possible total score of 10 , respectively. No significant differences in baseline characteristics were observed between the 2 groups, with the exception of age and total smoking amount.

\section{Continuous Abstinence Rates and 7-Day PP of Abstinence}

Table 2 shows the CAR and 7-day PP of abstinence (EC vs NG group). First, the efficacy of smoking cessation was assessed in terms of the CARs at 9 to 12 weeks and 9 to 24 weeks. The 9 - to 12 -week and 9- to 24-week CAR did not differ significantly between the 2 groups ( $45.3 \%$ vs $46.7 \%, P=.870$; $21.3 \%$ vs $28 \%, P=.344$ ) (Figure $3-\mathrm{A}$ ). Evaluation of a logistics model confirmed no significant difference after adjusting for confounding baseline factors (age and total smoking amount). Second, efficacy of smoking cessation was evaluated with regard to the 7-day $\mathrm{PP}$ of abstinence at 12 and 24 weeks. The 7-day PP of abstinence at week 12 was 
Table 1. Baseline Characteristics of the Study Subjects

\begin{tabular}{|c|c|c|c|c|}
\hline Characteristic & $\begin{array}{l}\text { All Subject } \\
(\mathrm{n}=150)\end{array}$ & $\begin{array}{l}\text { EC Group } \\
(\mathrm{n}=75)\end{array}$ & $\begin{array}{l}\text { NG Group } \\
(\mathrm{n}=75)\end{array}$ & $P$ Value* \\
\hline Age, years & $42.3 \pm 8.3$ & $44.0 \pm 7.8$ & $40.7 \pm 8.4$ & .014 \\
\hline Male sex, N (\%) & $150(100)$ & $75(100)$ & $75(100)$ & 1.000 \\
\hline \multicolumn{5}{|l|}{ Education level, N (\%) } \\
\hline$\leq$ High school & $91(60.7)$ & $51(68.0)$ & $40(53.3)$ & \multirow[t]{2}{*}{.066} \\
\hline$\geq$ College & $59(39.3)$ & $24(32.0)$ & $35(46.7)$ & \\
\hline \multicolumn{5}{|l|}{ Marriage status, N (\%) } \\
\hline Unmarried & $15(10.0)$ & $5(6.7)$ & $10(13.3)$ & \multirow[t]{2}{*}{.174} \\
\hline Married & $135(90.0)$ & $70(93.3)$ & $65(86.7)$ & \\
\hline Alcohol user, N (\%) & $107(71.3)$ & $55(73.3)$ & $52(69.3)$ & .588 \\
\hline Exercise, N (\%) & $82(54.7)$ & $45(60.0)$ & $37(49.3)$ & .189 \\
\hline Total smoking amount, pack-year & $21.56 \pm 9.71$ & $23.84 \pm 9.40$ & $19.28 \pm 9.54$ & .004 \\
\hline Cigarettes per day smoked, pack & $1.01 \pm 0.37$ & $1.05 \pm 0.37$ & $0.96 \pm 0.36$ & .161 \\
\hline Duration of smoking, years & $21.98 \pm 8.76$ & $23.26 \pm 7.60$ & $20.69 \pm 9.67$ & .072 \\
\hline Initial confidence about quitting smoking, score & $5.95 \pm 2.67$ & $6.12 \pm 2.57$ & $5.79 \pm 2.77$ & .447 \\
\hline FTND, score & $4.05 \pm 2.24$ & $4.00 \pm 2.22$ & $4.09 \pm 2.27$ & .799 \\
\hline \multicolumn{5}{|l|}{ Number of prior attempts to quit (\%) } \\
\hline None & $15(10.0)$ & $10(13.3)$ & $5(6.7)$ & \multirow[t]{3}{*}{.193} \\
\hline 1 & $31(20.7)$ & $12(16.0)$ & $19(25.3)$ & \\
\hline$\geq 2$ & $104(69.3)$ & $53(70.7)$ & $51(68.0)$ & \\
\hline
\end{tabular}

EC, electronic cigarette; NG, nicotine gum; FTND, Fagerström test for nicotine dependence.

${ }^{*} P$ values were calculated by independent $t$ test (for continuous variables) and $\chi^{2}$ test (for categorical variables).

$65.3 \%$ in the EC group and $66.7 \%$ in the NG group, showing no significant difference $(P=$ .863). At week 24 , it was $22.7 \%$ in the EC group and $29.3 \%$ in the NG group, again showing no significant difference $(P=.352)$. In addition, there was still no significant difference after adjusting for confounding factors.

\section{Reduction in Smoking Rate and Amount between Both Groups}

At baseline, the ranges of the number of cigarettes smoked per day between the 2 groups (EC vs NG group) were $20.95 \pm 7.41$ (range, 10 to 40 ) and $19.27 \pm 7.22$ (range, 10 to 40 ). At 12 weeks, there was no difference in the proportion of subjects with a reduced smoking rate and reduction in the mean number of cigarettes consumed in a day between the 2 groups. However, at 24 weeks, the proportion of subjects who had accomplished smoking reduction was significantly higher in the EC group, namely, $41.3 \%$ compared with $25.3 \%$ in the NG group $(P=.038)$. Both groups showed an approximate mean reduction of 6.6 cigarettes per day (EC group, $6.55 \pm 2.87$ [range 2 to 15]; NG group, $6.60 \pm 3.75$ [range, 5 to 20]) (Table 3 ).

\section{Tolerability}

In our study, there were no serious AEs. The percentage of subjects who experienced AEs was significantly lower in the EC group than in the NG

Table 2. Comparison of Continuous Abstinence Rate and 7-Day Point Prevalence of Abstinence in the Two Groups

\begin{tabular}{lcccc}
\hline Outcome & EC Group & NG Group & Crude $P$ Value & Adjusted $P$ Value $^{*}$ \\
\hline CAR at 9 to 12 week, (\%) & 45.3 & 46.7 & .870 & .947 \\
CAR at 9 to 24 week, (\%) & 21.3 & 28.0 & .344 & .291 \\
7-day PP of abstinence at 12 week, (\%) & 65.3 & 66.7 & .863 & .602 \\
7-day PP of abstinence at 24 week, (\%) & 22.7 & 29.3 & .352 & .365 \\
\hline
\end{tabular}

EC, electronic cigarette; NG, nicotine gum; CAR, continuous abstinence rates; PP, point prevalence.

*Adjusted for confounding baseline factors, such as age and total smoking amount, i.e., pack-year smoked. 
Figure 3. Efficacy of smoking cessation according to interventions, electronic cigarette versus nicotine gum. (A) Continuous abstinence rates at 9- to 12-weeks and 9- to 24-weeks; and (B) 7-day PP of abstinence. NS, nonsignificant; $P P$, point prevalence.

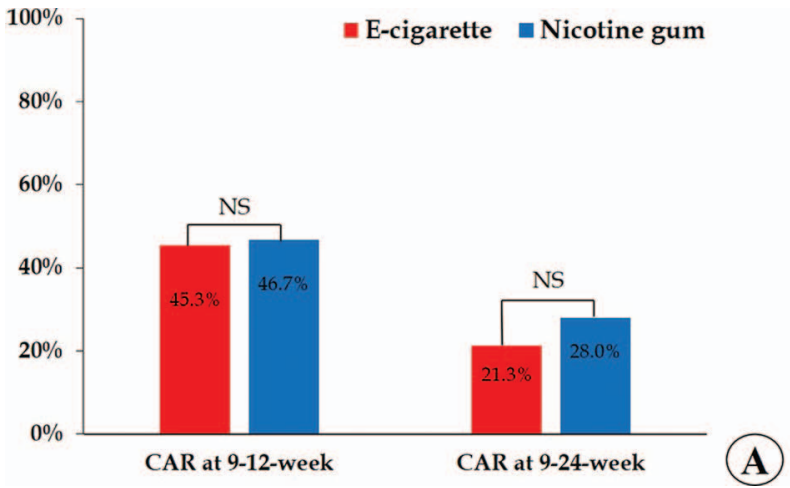

group $(6.7 \%$ vs $17.3 \%, P=.044)$. The most common AEs in both groups were oral pain, cough, dry mouth, headache, and nausea/vomiting in both groups (Table 4). No significant differences were observed between the 2 groups in the prevalence of most AEs. However, nausea and vomiting were relatively more frequent in the NG group than in the EC group $(P=.034)$. The AEs in this study were of mild-to-moderate intensity and none led to withdrawal from the study.

\section{Discussion}

The purpose of this study was to investigate the effect of e-cigarettes on smoking cessation and to compare them with those of nicotine gum, a welldocumented NRT. There was no significant difference in the effect of e-cigarettes over the course of 24 weeks compared with that of the nicotine gum. In addition, e-cigarettes were associated with the higher proportion of participants with smoking reduction and were more tolerable than nicotine gum.

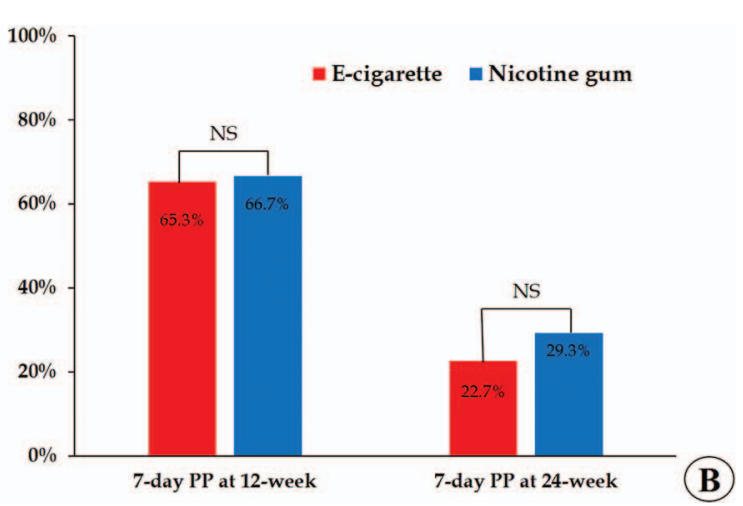

In our study, the 9- to 12-week and 9- to 24week CARs of the EC group were considerably higher than those reported previously $(45.3 \%$ vs $13.1 \%$ and $21.3 \%$ vs $7.3 \%$, respectively). ${ }^{12}$ In addition, the values of the 7-day PP of abstinence at 12 and 24 weeks were both higher than those reported previously for e-cigarettes and nicotine inhalers. ${ }^{15,16}$ Possible explanations are that NRTs are more likely to result in successful smoking cessation in males than in females and that the decline in the desire to smoke is significantly greater in the NRT group compared with placebo group. ${ }^{16,17}$ It is believed that the positive environment of the company's antismoking campaign also contributed to the present study's successful results.

In the present study, $41.3 \%$ of the EC group achieved a mean reduction of approximate 6.6 cigarettes per day, thereby demonstrating a significant harm reduction similar to the results of previous studies. As already noted, previous studies on nicotine inhalers, which have a mechanism similar to that of e-cigarettes, have demonstrated their effec-

Table 3. Reduction in Smoking Rate and Amount between the Two Groups

\begin{tabular}{lccc}
\hline Outcome & EC Group & NG Group & $P$ Value* \\
\hline 12 week & & & \\
$\quad$ Proportion of subjects with reduced daily cigarettes, N (\%) & $16(21.3)$ & $15(20.0)$ & .840 \\
$\quad \begin{array}{l}\text { Reduction in the mean number of cigarettes consumed in a day } \\
24 \text { week }\end{array}$ & $11.06 \pm 7.03$ & $12.60 \pm 5.65$ & .509 \\
$\quad \begin{array}{l}\text { Proportion of subjects with reduced daily cigarettes, N (\%) } \\
\text { Reduction in the mean number of cigarettes consumed in a day }\end{array}$ & $31(41.3)$ & $19(25.3)$ & .038 \\
\hline
\end{tabular}

EC, electronic cigarette; NG, nicotine gum.

${ }^{*} P$ values were calculated by independent $t$ test (for continuous variables) and $\chi^{2}$ test (for categorical variables). 
Table 4. Frequency of Adverse Events

\begin{tabular}{lccc}
\hline AE, N (\%) & EC Group & NG Group & $P$ Value \\
\hline Subjects with any AEs* & $5(6.7)$ & $13(17.3)$ & .044 \\
Total AEs & $9(100)$ & $27(10)$ & \\
Sore throat $^{\dagger}$ & - & $2(7.4 \%)$ & .497 \\
Oral pain $^{\dagger}$ & $2(22.2)$ & $5(18.5)$ & .442 \\
Cough $^{\dagger}$ & $3(33.3)$ & $3(11.1)$ & 1.000 \\
Dry mouth $^{\dagger}$ & $2(22.2)$ & $2(7.4)$ & 1.000 \\
Oral ulcer $^{\dagger}$ & - & - & - \\
Dizziness $^{\dagger}$ & - & $5(18.5)$ & .058 \\
Headache $^{\dagger}$ & $1(11.1)$ & $2(7.4)$ & 1.000 \\
Nausea or vomiting $^{\dagger}$ & $1(11.1)$ & $8(29.6)$ & .034 \\
Others $^{\dagger}$ & - & - & - \\
\hline
\end{tabular}

AEs, adverse events; EC, electronic cigarette; NG, nicotine gum.

${ }^{*} P$ value was calculated using the $\chi^{2}$ test.

${ }^{\dagger} P$ value was calculated using the Fisher-Freeman-Halton extension of Fisher's probability test.

tiveness for smoking cessation. ${ }^{16,18-20}$ Our e-cigarette results are consistent in this regard. Some researchers have questioned the need for e-cigarettes as a treatment for nicotine dependence, as nicotine inhalers are already available. However, whereas the 2 products have similar mechanisms of action, e-cigarettes have the advantage of giving the smoker the sensation of actually smoking. ${ }^{21} \mathrm{We}$ believe that this aspect of e-cigarettes provides a fundamental additional advantage over the previously introduced NRTs (patches, lozenges, gums, tablets, and inhalers) in this regard.

E-cigarettes are not yet standardized and so the effects can vary among products. The product used in this study does not produce tar or carbon monoxide and has been reported by the WHO to have minimal formaldehyde and acetaldehyde levels similar to those found in nature. According to a study done in New Zealand, e-cigarettes do not produce polycyclic aromatic hydrocarbon carcinogens, which are found in regular cigarettes. ${ }^{22}$

Another previous study found no significant differences in the side effects of e-cigarette and nicotine patches. ${ }^{12}$ In contrast, in the present study, those who used e-cigarettes had significantly fewer side effects than those who used nicotine gum; the reported side effects, moreover, were mild and well-tolerated, and none of the subjects needed to stop using e-cigarettes because of them. The most frequently reported side effects were cough and dry mouth, which are consistent with previous reports. ${ }^{2}$
There may be some drawbacks to e-cigarettes. Concerns have been raised regarding the possibility that e-cigarettes might act as a gateway, leading adolescents to cigarette smoking. ${ }^{3,7}$ There are also other concerns that, for example, users will develop e-cigarette dependency or use both e-cigarettes and combustible cigarettes. ${ }^{5,23,24}$ In this light, a better solution may be to identify e-cigarettes as a treatment tool and control their use through regulatory policies and physician prescriptions rather than making them freely available on the commercial market.

The strength of this study is that it is the first study, to our knowledge, that has evaluated the effect of e-cigarettes on smoking cessation compared with a well-documented NRT (nicotine gum) in Korean males. Another strength of our study was the measurement of carbon monoxide and urine cotinine levels in subjects who reported smoking cessation; such measures are more objective than self-reports. In addition, 2 measurements supported self-reported 9- to 12-week and 9- to 24-week CAR. There were no statistically significant deviations. However, the present study also had several limitations. First, the study sample size was relatively small and, therefore, our study may not have adequate power to detect the differences of results related to smoking cessation between the 2 groups. In addition, this was a single-center trial. Thus, further multicenter and large-scale studies are needed to confirm these findings. The second limitation of the present study was the significantly higher number of pretrial drop-outs in the NG group than in the EC group $(P=.012)$. However, we assume that the higher rate of dropout in the NG group is because participants were hoping to receive e-cigarettes. As we considered all the dropouts as failures in smoking cessation in the intention-to-treat analyses, this might have led to underestimation of the smoking-cessation success rate in the NG group relative to the EC group. Third, we were unable to evaluate the craving, withdrawal, or reinforcing effects in the EC group. Finally, as this study involved only with Korean males, its results may not be readily generalizable to other populations.

Despite this study's limitations, it can be used as a basis for further research on e-cigarettes and their effects. Also, additional research into the long-term safety of e-cigarettes is necessary before such devices can be widely used as smoking-cessation tools. 
In conclusion, the results of our study suggest that the efficacy of the e-cigarette, a novel method of NRT, for smoking cessation is not inferior to that of nicotine gum among Korean male smokers motivated to quit smoking. The e-cigarette generally was well tolerated, with only mild-to-moderate AEs, including cough and dry mouth. Therefore, based on our study, e-cigarettes may be considered as a useful NRT for smoking cessation. A future large-scale prospective randomized controlled trial is necessary to clarify our findings.

To see this article online, please go to: bttp://jabfm.org/content/ 32/4/567.full.

\section{References}

1. Vansickel AR, Eissenberg T. Electronic cigarettes: effective nicotine delivery after acute administration. Nicotine Tob Res 2013;15:267-70.

2. Polosa R, Caponnetto P, Morjaria JB, Papale G, Campagna D, Russo C. Effect of an electronic nicotine delivery device (e-Cigarette) on smoking reduction and cessation: a prospective 6-month pilot study. BMC Public Health 11:786, 2011.

3. Yamin CK, Bitton A, Bates DW. E-cigarettes: a rapidly growing Internet phenomenon. Ann Intern Med 2010;153:607-9.

4. WHO Study Group on Tobacco Product Regulation. Report on the scientific basis of tobacco product regulation: third report of a WHO Study Group. World Health Organ Tech Rep Ser 2009:1-41, back cover.

5. Etter JF. Electronic cigarettes: a survey of users. BMC Public Health 2010;10:231.

6. Fiore MC, Schroeder SA, Baker TB. Smoke, the chief killer-strategies for targeting combustible tobacco use. N Engl J Med 2014;370:297-9.

7. Lee S, Kimm H, Yun JE, Jee SH. Public health challenges of electronic cigarettes in South Korea. J Prev Med Public Health 2011;44:235-41.

8. Farsalinos KE, Spyrou A, Stefopoulos C, Tsimopoulou K, Kourkoveli P, Tsiapras D, et al. Nicotine absorption from electronic cigarette use: comparison between experienced consumers (vapers) and naive users (smokers). Sci Rep 2015;5:11269.

9. Manzoli L, Flacco ME, Fiore M, La Vecchia C, Marzuillo C, Gualano MR, et al. Electronic cigarettes efficacy and safety at 12 months: cohort study. PLoS One 2015;10:e0129443.
10. Pacifici R, Pichini S, Graziano S, Pellegrini M, Massaro G, Beatrice F. Successful nicotine intake in medical assisted use of e-cigarettes: a pilot study. Int J Environ Res Public Health 2015;12:7638-46.

11. Sutfin EL, Reboussin BA, Debinski B, Wagoner KG, Spangler J, Wolfson M. The impact of trying electronic cigarettes on cigarette smoking by college students: a prospective analysis. Am J Public Health 2015;105:e83-9.

12. Bullen C, Howe C, Laugesen M, et al. Electronic cigarettes for smoking cessation: a randomised controlled trial. Lancet 2013;382:1629-37.

13. Morris K. Revising the Declaration of Helsinki. Lancet 2013;381:1889-90.

14. Barth I, Krafft H, Weber G, Keller-Stanislawski B, Cichutek K. Good clinical practice in the European Union. Hum Gene Ther 2008;19:441-2.

15. Siegel MB, Tanwar KL, Wood KS. Electronic cigarettes as a smoking-cessation: tool results from an online survey. Am J Prev Med 2011;40:472-5.

16. Rennard SI, Glover ED, Leischow S, et al. Efficacy of the nicotine inhaler in smoking reduction: a double-blind, randomized trial. Nicotine Tob Res 2006; 8:555-64.

17. Shiffman S, Fant RV, Buchhalter AR, Gitchell JG, Henningfield JE. Nicotine delivery systems. Expert Opin Drug Deliv 2005;2:563-77.

18. Schneider NG, Olmstead RE, Franzon MA, Lunell E. The nicotine inhaler: clinical pharmacokinetics and comparison with other nicotine treatments. Clin Pharmacokinet 2001;40:661-84.

19. Caldwell B, Dickson S, Burgess C, Siebers R, Mala $\mathrm{S}$, Parkes A, et al. A pilot study of nicotine delivery to smokers from a metered-dose inhaler. Nicotine Tob Res 2009;11:342-7.

20. Kralikova E, Kozak JT, Rasmussen T, Gustavsson G, Le Houezec J. Smoking cessation or reduction with nicotine replacement therapy: a placebo-controlled double blind trial with nicotine gum and inhaler. BMC Public Health 2009;9:433.

21. Kralikova E, Kubatova S, Truneckova K, Kmetova A, Hajek P. The electronic cigarette: what proportion of smokers have tried it and how many use it regularly? Addiction 2012;107:1528-9.

22. Laugesen M. Second Safety Report on the Ruyan $®$ e-cigarette. Cell 2008;27:4375.

23. Etter JF, Bullen C. Electronic cigarette: users profile, utilization, satisfaction and perceived efficacy. Addiction 2011;106:2017-28.

24. Cho JH, Shin E, Moon SS. Electronic-cigarette smoking experience among adolescents. J Adolesc Health 2011;49:542-6. 